\title{
A National Study of Community Health Centers' Readiness to Address COVID-19
}

\author{
April Joy Damian, PhD, MSc, Melanie Gonzalez, MA, May Oo, MPH, and \\ Daren Anderson, MD
}

Background: The coronavirus disease 2019 (COVID-19) outbreak poses serious health risks, particularly for older adults and persons with underlying chronic medical conditions. Community health centers (CHCs) serve as the patient medical home for populations that are disproportionately more susceptible to COVID-19; yet, there is a lack of understanding of current efforts in place by CHCs to prepare for and respond to the pandemic.

Methods: We used a comprehensive cross-sectional survey and focus groups with health care personnel to understand the needs and current efforts in place by CHCs, and we derived themes from the focus group data.

Results: Survey respondents ( $n=234 ; 19 \%$ response rate) identified COVID-19 infection prevention and control (76\%), safety precautions (72\%), and screening, diagnostic testing, and management of patients $(66 \%)$ as major educational needs. Focus group findings $(n=39)$ highlighted 5 key themes relevant to readiness: leadership, resources, workforce capacity, communication, and formal policies and procedures.

Conclusion: The COVID-19 pandemic has exacerbated long-standing CHC capacity issues making it challenging for them to adequately respond to the outbreak. Policies promoting greater investment in CHCs may strengthen them to better meet the needs of the most vulnerable members of society, and thereby help flatten the curve. (J Am Board Fam Med 2021;34:S85-S94.)

Keywords: Community Health Centers, Community Medicine, COVID-19, Cross-Sectional Studies, Focus Groups, Health Workforce, Leadership, Pandemics, Patient-Centered Care, Public Health, Surveys and Questionnaires

\section{Introduction}

On January 30, 2020, the World Health Organization (WHO) described the coronavirus disease 2019 (COVID-19) outbreak as a public health emergency of international concern. ${ }^{1}$ Less than 6 weeks later, WHO publicly characterized the novel respiratory disease as a pandemic. ${ }^{2}$ The US federal administration's actions followed suit by initially declaring a public health emergency on January 31, 2020, then on March 13, 2020, officially declaring a national emergency. Although the Centers for Disease Control and Prevention (CDC) has noted that the United States is currently in the initiation phases with some states now

This article was externally peer reviewed.

Submitted 22 April 2020; revised 24 June 2020; accepted 27 June 2020.

From the Weitzman Institute, Middletown, CT.

Funding: None.

Conflict of interest: None.

Corresponding author: April Joy Damian, PhD, MSc, Weitzman Institute, Community Health Center, Inc., 19 Grand Street, Middletown, CT 06457 (E-mail: adamian2@ jhu.edu). in the acceleration phase, emerging evidence shows that some people are at higher risk than others of becoming very ill from COVID-19, including older adults and persons with chronic medical conditions such as heart disease, diabetes, and lung disease. ${ }^{3}$

For more than 50 years, community health centers (CHCs) in the United States, through funding from the US Health Resources and Services Administration, have functioned as the nation's primary care backbone for the medically underserved and have been at the forefront of serving the most vulnerable members of society. CHCs provide essential medical, behavioral health, and dental care to patients who disproportionally suffer from chronic health challenges and have poorer health outcomes. ${ }^{4}$ Twenty-eight million people nationwide (1 in 12) rely on CHCs for affordable and accessible primary care, including 1 in 5 rural residents, 9 out of 10 persons living in poverty or near poverty, more than 1.2 million persons experiencing homelessness, and more than 385,000 veterans. ${ }^{5}$ Moreover, CHC patients are more likely to be unemployed and 
uninsured compared with the general low-income population in the United States. ${ }^{4}$

In addition to meeting the comprehensive primary care needs of the country's medically underserved populations, $\mathrm{CHCs}$ play a vital role in supporting the US public health system and have demonstrated success in addressing other public health challenges. For example, in 2018, as part of the nation's effort to end the HIV epidemic, health centers provided more than 2.4 million HIV tests to more than 2 million patients and treated 1 in 6 patients diagnosed with HIV nationally. In the same year, CHCs screened and identified nearly 1.1 million people for substance use disorder and have, since 2017, provided medication-assisted treatment to nearly 95,000 patients nationwide, an increase of $143 \% .^{5}$

Nonetheless, even in the midst of current emergency preparedness and response efforts to the COVID-19 pandemic at the local, state, and national levels, there is a lack of understanding about the extent to which these efforts are meeting the needs of safety-net practices, their workforce, and, subsequently, their patients who are most susceptible to COVID-19 and its negative health and social-related sequelae. In addition, it is important to frame this work in the baseline realities of safetynet practices by recognizing that CHCs were already under-resourced even before the current pandemic, which exacerbates the current stressors. The purpose of this mixed-methods assessment was to understand CHCs' needs and readiness to address COVID-19. To our knowledge, this is the first and only national, multisite study of its kind.

\section{Methods}

\section{Study Design}

We used a sequential explanatory mixed-methods approach to understand the needs and current efforts in place by primary care staff and practices across the United States working to effectively prepare for COVID-19. Findings from the baseline cross-sectional survey were subsequently used to develop the interview guide for the focus groups.

\section{Survey Development}

We developed a web-based, cross-sectional survey regarding organizational and individual staff needs and best practices currently in place to prepare for and prevent spread of COVID-19 (Appendix 1).
Four items were selected for the survey. These items directly reflect the domains of interest for health care professionals that were outlined on the CDC resource page at the time that this study was being designed. ${ }^{6}$ We subsequently pilot tested the survey with 6 administrators and clinicians at Community Health Center, Inc. (CHCI) who were representative of the target population to provide input on the initial survey, particularly the wording, ordering, and administrative burden of completing the survey. Improvements were made to the survey and were reflected in the finalized administered survey. Registrants had the opportunity to complete the survey on SurveyMonkey beginning on March 3, 2020 through March 6, 2020.

\section{Survey Administration}

The survey was administered to 1259 health workers in the United States who registered for a special Weitzman Institute Project ECHO. Registrants represented 730 health organizations from 49 states (Idaho not represented), Washington D.C., Puerto Rico, and Guam. Of those registrants invited to participate, 240 people completed this survey, yielding a response rate of $19 \%$.

\section{Focus Group Schedule Development}

The last question of the survey we administered asked respondents if they would be willing to participate in a focus group regarding $\mathrm{CHC}$ response to COVID-19. Of the 240 people who completed the survey, 131 expressed interest in participating in the focus groups. These 131 survey respondents were subsequently invited via e-mail to sign up for a focus group to provide more in-depth responses regarding organizational and individual readiness. Participants were offered to the opportunity to attend 1 of 7 virtual focus groups using the Zoom videoconferencing platform. We enrolled a maximum of 10 participants per focus group.

\section{Focus Group Administration}

Of those who completed the survey, a convenience sample of 39 participants agreed to attend 1 of a series of hour-long focus groups held within 1 week of the initial survey being administered. Three of the 39 participants participated in the focus group but did not complete a survey. These 3 individuals were forwarded the request from a colleague within their organization who was invited to the focus group. Focus groups have been identified as an 
economical, fast, and efficient method for obtaining data from multiple participants, thereby potentially increasing the overall number of participants in a given qualitative study. ${ }^{7,8}$ The focus groups covered 3 domains based on a priori discussions with key informants (eg, CHC providers, administrators): (1) organizational and staff strengths and gaps in COVID-19 preparedness, (2) psychosocial impact of COVID-19 on health workers and patients, and (3) role of technology, particularly telehealth (Appendix 2). The focus group facilitator closely followed the interview protocol in an effort to minimize potential interviewer bias. All focus group sessions were audio recorded. Focus group attendance ranged from 5 to 7 participants in each session. The current report focuses on data derived from the first 2 domains.

\section{Data Analysis}

The research team applied descriptive statistics to analyze the survey data and determine frequency of endorsing the needs outlined in the survey. The research team subsequently applied the transcriptbased analysis approach to the focus group data, which represents the most rigorous model of analyzing the data ${ }^{9} ; 2$ trained coders independently applied an iterative coding process to analyze the interviews and derive themes using the constant comparative method. ${ }^{10}$ Consensus meetings were used to come to agreement on final codes, definitions, and exemplars to be included. This study was approved by the CHCI Institutional Review Board.

\section{Results}

We observed a $19 \%$ response rate, as the survey was completed by 240 primary care employees from 201 organizations across 44 states, the District of Columbia, and Puerto Rico (Table 1). Six participants were excluded from the initial survey due to incomplete data, leaving 234 participants in the study. The majority of respondents (59\%) held administrative leadership roles at their respective agencies, and the remainder of the participants served in patient-facing roles as physicians $(8 \%)$, nurses (13\%), and medical support staff (20\%). More than a third $(38 \%)$ of the participants came from practices in the Northeast region of the country, with the rest of respondents coming from the West (19\%), South (19\%), and Midwest (23\%) regions, and minor representation from Puerto Rico (1\%).

Findings from the survey revealed that the top educational and resource needs endorsed by participants according to their respective job titles include guidance regarding COVID-19 infection prevention and control (76\%), safety precautions for patient-facing health care personnel (72\%), and screening, diagnostic testing, and management of patients (66\%). In addition, 199 out of 234 respondents $(85 \%)$ indicated the need for safety planning checklists, including policies and procedures for emergency preparedness because their respective practices either do not currently have these plans in place or participants are largely unaware of such resources if they do exist (Table 2). Analysis of the focus group data revealed 5 overarching themes

Table 1. Characteristics of Participants Who Completed Surveys Between March 3, 2020 and March 6, 2020 and Who Participated in Focus Groups Between March 10, 2020 and March 17, 2020

\begin{tabular}{lcr}
\hline & Survey Respondents $(\mathrm{n}=234)$ & Focus Group Respondents $(\mathrm{n}=39)$ \\
\hline Role & $\mathrm{n}(\%)$ & $\mathrm{n}(\%)$ \\
Administrative leadership & & $18(46 \%)$ \\
Physician & $139(59 \%)$ & $9(23 \%)$ \\
Nurse & $19(8 \%)$ & $4(10 \%)$ \\
Medical support staff & $30(13 \%)$ & $8(21 \%)$ \\
Region & $46(20 \%)$ & $13(33 \%)$ \\
Northeast & & $12(31 \%)$ \\
Midwest & $90(38 \%)$ & $5(13 \%)$ \\
South & $53(23 \%)$ & $8(20 \%)$ \\
West & $44(19 \%)$ & $1(3 \%)$ \\
Puerto Rico & $44(19 \%)$ & $3(1 \%)$
\end{tabular}


Table 2. Top Educational and Resource Needs Endorsed by Participants Who Completed Surveys Between March 3, 2020 and March 6, 2020

\begin{tabular}{|c|c|c|c|c|}
\hline & \multicolumn{4}{|c|}{ Survey Respondents $(\mathrm{n}=234)$} \\
\hline & $\begin{array}{l}\text { COVID-19 infec- } \\
\text { tion prevention } \\
\text { and control } \\
\mathrm{n}(\%)\end{array}$ & $\begin{array}{l}\text { Screening, diagnostic } \\
\text { testing, and management } \\
\text { of patients } \\
\mathrm{n}(\%)\end{array}$ & $\begin{array}{l}\text { Safety precautions for } \\
\text { patient-facing health } \\
\text { care personnel } \\
\mathrm{n}(\%)\end{array}$ & $\begin{array}{l}\text { Safety planning checklists, including } \\
\text { policies and procedures for emergency } \\
\text { preparedness } \\
\mathrm{n}(\%)\end{array}$ \\
\hline \multicolumn{5}{|l|}{ Role } \\
\hline $\begin{array}{l}\text { Administrative } \\
\text { leadership }\end{array}$ & $107(46 \%)$ & $90(38 \%)$ & $99(42 \%)$ & $116(50 \%)$ \\
\hline Physician & $15(6 \%)$ & $14(6 \%)$ & $14(6 \%)$ & $17(7 \%)$ \\
\hline Nurse & $22(9 \%)$ & $21(9 \%)$ & $18(8 \%)$ & $24(10 \%)$ \\
\hline Support staff & $35(15 \%)$ & $30(13 \%)$ & $37(16 \%)$ & $42(18 \%)$ \\
\hline \multicolumn{5}{|l|}{ Region } \\
\hline Northeast & $72(31 \%)$ & $57(24 \%)$ & $67(30 \%)$ & $77(33 \%)$ \\
\hline Midwest & $41(18 \%)$ & $38(16 \%)$ & $37(16 \%)$ & $44(19 \%)$ \\
\hline South & $32(14 \%)$ & $29(12 \%)$ & $32(14 \%)$ & $36(15 \%)$ \\
\hline West & $33(14 \%)$ & $30(13 \%)$ & $29(12 \%)$ & $39(17 \%)$ \\
\hline Puerto Rico & $1(0.4 \%)$ & $1(0.4 \%)$ & $3(1 \%)$ & $3(1 \%)$ \\
\hline
\end{tabular}

relevant to foundational aspects of readiness including leadership, resources, workforce capacity, communication, and formal policies and procedures (Table 3).

\section{Leadership}

Leadership, defined as providing general oversight of the practice and/or team(s), was described as an important aspect of organizational and individual readiness. Some respondents noted that decision making was challenging when health center leaders received conflicting guidance and recommendations provided by leading government and public health authorities. In addition, there were multiple descriptions about the need for unity and alignment among senior leadership, including among administrative and clinical senior leaders. Conversely, there were concerns raised about both existing and potential disagreement among leadership and its impact on health center decision making during public health crises such as the current pandemic. For example, participants described competing priorities with regards to health centers' bottom line and financial well-being, with the health and safety of staff and patients. Others also commented on the need for leadership to promote clarity in defining roles of different health care team members.

\section{Resources}

Across respondents, there was resounding concern over CHCs having limited resources, specifically testing supplies, personal protective equipment (PPE), and negative pressure rooms. Respondents discussed not being able to meet demand from patients for COVID-19 testing. Similarly, respondents admitted to challenges with staff not having access to N95 face masks but also not having undergone fit testing or training on proper fitting, wearing, and removal of masks because this was not deemed to be a priority until the current situation arose. In addition, respondents expressed challenges with government support and issuing of supplies not reaching CHCs. Lastly, ethical issues of staff stealing health center supplies for personal use was also raised, along with the ramifications that these actions have on the health and safety of health center staff and patients.

\section{Workforce Capacity}

Participants noted the acute and chronic challenge of workforce capacity, defined as sufficient staffing levels to carry out necessary work processes. Comments were made noting that CHCs are having to quickly focus on and prioritize COVID-19 but are having to do so with limited staffing capacity. Respondents noted how the current pandemic has contributed to workforce burnout by adding to the already demanding daily tasks that CHC staff have, particularly in serving large patient panels in the midst of other health center closures and medically complex patients with limited resources. In addition, participants also pointed out the 
Table 3. Focus Group Key Themes Related to Organizational Readiness to Address COVID-19

Theme Sample Quotes

Leadership One of the barriers is conflicting guidance between Delaware Department of Health, CDC, and World Health Organization, which creates internal conflicts since some leaders will follow one, and the others follow another. (Director of Quality Improvement, Delaware)

Another barrier is with our finance administrative folks who think from an economic standpoint and if we need to close clinics and let people work from home, they are thinking about a loss of revenue, which is in opposition with the clinical people who are more concerned about the safety of our staffs and patients. (Chief Medical Officer, Illinois)

Definitely challenging for us to make sure that leadership is on the same page because our executive directors and medical directors have had different opinions at time so it is really important for us to come together quickly making sure we are considering the health and well-being of our staffs and patients, and for our financial results, how much to charge for telehealth visit. (Practice Manager, Ohio)

Resources Everybody wants testing and we don't have the capacity to test everybody. That's my biggest concern. Because we were in the rural side of the state, we just got the testing materials this week. The fact is everyone with a sniffle wants to get it tested and we don't have the capacity to do that. (Director of Training and Compliance, Washington)

One of the main issues we are having is geographic spread we have across the state. We have clinics that are very small, 2 to 3 rooms and one solo provider or clinician. If we have 1 or 2 to the patients that need to be isolated, that creates a significant reduction in the way that we care for patients. (Vice President of Clinical Affairs, New Mexico)

The biggest problem we are having is not having enough PPE. We are running out of masks, and I also know that we are running out of all of the necessary wipes and everything although we have a plan to go to like bleaching water solution for that. Even though the California governor is issuing a million supplies, they are not falling through the system to get to community health centers. (Chief Medical Officer, California)

We noticed some of our masks disappear, so we have to put out a notice that even though we know you want to protect yourself and your family, you cannot take health center's properties and we will take action if anybody does that. (Primary Care Provider, New York)

Workforce I think it is a lot more stressed for us because we are able to see the deficits that we have in our system and those capacity deficits are rapidly progressing. All of us here are very stressed out and we are adding that on top of the things that we do already every day. (Clinical Educator, Illinois)

The other complexity is another health center near us has a significant number of the homeless population in the community, and that center closed in December. So, we still have many of those folks that have not established primary care elsewhere and then they may be showing up in our lobby or at our doorway requesting assistance without having any other route. (Director of Nursing, Michigan)

In reality, most of my NPs have babies and multiple kids. When we start thinking about quarantine and women dominated workforce like I have, it's a huge worry for me....And other organizations, they are just laying workers off and I know I have lots of people who need their pay check and so what are the consequences, how do we keep the money flow so that my employees don't become part of the economic ripple effect, which ends up impacting the personal life. (Chief Executive Officer, Ohio)

Communication We never really get communication out to our staffs in a desired time frame. We have a lot of providers, a lot of staff, taking a word of media, from family and communities, rather than realizing that we are the experts in health care. (Director of Training and Compliance, Washington)

We implemented basically_for the administration we do a daily brief update on COVID-19, so we are able to make sure that we are standardizing every site here. So, we might have a clinical center director who really goes into details and someone from another site who doesn't have a clinical background, they might give different messages, so we are using our daily brief to actually standardize the message to everyone across the organization, and I think that's been very helpful. (Chief Executive Officer, Ohio)

Our role as an educator is pretty massive in educating our communities, not all of them are medically educated and so they depend on us for COVID-19 information. We are going to look at how we reach out to our communities differently in the future as well. (Community Health Worker, Michigan)

I think communication to the patients-some folks are not understanding fully, with what to do. They come in and they say that they have been in contact with someone who tested positive, and they have no symptoms and they are confused where they can get tested. So, it has been difficult to try to explain to the folks the processes, and I hope that there is the larger testing system coming in place that we can all use. I think that is the biggest piece-making sure that we are educated and the public is educated as well, so giving that consistency across the board has been a challenge. (Director of Facilities, Illinois)

Formal

policies and

We have a great big binder that has everything everyone wants to know, and quite honestly it didn't help much at all in the preparation for this. So, having plans and policies sit on the shelves, I think we probably did ours, you know, 10 years ago, and you know with everything else we are all working on, it becomes less of the priority. So that we have to plan, which really is not much help, but we have to do everything from inventory and find out where all our supplies were, start pretty much from scratch. (Director of Clinical Best Practice and Education, New York)

I am really struggling to find a written triage protocol to help providers and nurses decide in a situation like this. It seems like we are having to update our policies daily because the situation continues to change. And we also have a hard time pinning down the policies about testing. As of last week, we didn't have any commercially available testing, so we referred and directed anyone to the health department. Today, we got a phone call from the Texas health department that said not to send anyone to the health department. It's like every 2 days, we need to rewrite our protocols. (Associate Medical Director, Texas)

Starting next week, in order to keep our medical staff and other patients healthy, we have established a triage unit outside the buildings to detect any symptoms before they get to the regular population. (Health Educator, Puerto Rico)

We are not doing as many face-to-face visits, and employees are going to do home visits. So, we are looking at combined pharmacy delivering meds, home visits, and having maybe medical assistants or community outreach workers going to some of the high need people who don't have smartphone capability. (Chief Executive Officer, Georgia)

CDC, Centers for Disease Control and Prevention; PPE, personal protective equipment; NPs, nurse practitioners. 
workforce shortage and the subsequent strain on the remaining health care workforce when colleagues have to handle personal responsibilities.

\section{Communication}

There were a range of responses regarding level of readiness as well as needs around communication, defined by exchange of information. Several respondents described having comprehensive communication strategies across their respective practices that involve multiple modalities such as an internal command center, e-mail, and text messaging. In contrast, others admitted to major gaps in their internal communications and challenges with ensuring real-time dissemination of accurate information to both staff and patients. Respondents discussed the challenges of competing with and overcoming misinformation that is spread through mass media, which respondents have found to invoke fear, panic, and confusion among their patients, as well as determining how to communicate with hard-to-reach patient populations such as the elderly, who may be less technologically savvy. In other words, respondents agreed that there is not only a dire need to improve and ensure staff have a means of rapidly communicating with each other, there is also a shared sense of responsibility to be able to communicate with patients and communities to ensure they are well informed and equipped with timely and accurate information.

\section{Formal Policies and Procedures}

Similar to the comments regarding communications, respondents described varying degrees of readiness to implement and execute policies and procedures specific to the current pandemic. Although respondents for the most part noted having emergency preparedness protocols in place, they described major challenges in complying with a standard set of policies and procedures. Limited supplies made it difficult to be compliant with protocols; furthermore, the rapidly evolving nature of the current pandemic and the accompanying changes in guidance provided by government and public health authorities makes it difficult for CHCs to implement the most appropriate protocols. Nonetheless, some respondents provided details about how their respective practices have risen to the present-day challenge and implemented new procedures in response to COVID-19. Respondents shared some examples of additional safety precautions and alternatives for meeting demand, including triaging patients outside the clinical buildings as well as leveraging community health workers to conduct home visits particularly to high-need, hard-to-reach patients.

\section{Discussion and Conclusion}

Findings from both the survey and focus groups highlight the significant need for knowledge and capacity development, especially in the area of infection control policies and procedure. Although respondents noted a need for clinical information about testing and management of patients, their responses suggest an even greater need for guidance on carrying out proper infection control protocols. One prior study assessing readiness to tackle an influenza pandemic found that most health centers lacked the capacity to effectively implement needed infection control policies. ${ }^{11}$

For the current pandemic, it is unclear, based on these findings, whether the problem is a lack of appropriate policies and procedures or a lack of expertise and knowledge on how to properly implement and follow them. Eighty-five percent of respondents endorsed a need for tools such as safety checklists and formal policies to follow. However, focus group participants suggested that the need was less about having the policies and more about limited expertise or preparation to follow them. One respondent noted that fit testing for N95 masks had never been done, despite it being mandated in their policy. This finding is consistent with a study conducted before the COVID-19 pandemic that found that CHCs were less likely than local health departments to have engaged in actual preparedness planning, such as conducting drills. ${ }^{12}$ Immediate attention is needed to address these deficiencies, in the form of educational tools and resources on proper infection control procedures.

In addition to the more universal concerns about lack of testing and PPE, these findings highlight the special challenge posed by lack of staffing resources in CHCs. Recruiting and retaining staff in rural and other medically underserved practice locations has burdened health centers long before the pandemic. ${ }^{13}$ Conversely, COVID-19 will inordinately affect the most vulnerable segments of the population, including those with the fewest resources to deal with its medical and financial consequences. Low staffing ratios and unfilled positions 
leave little in the way of "surge capacity" needed to address the expected increase in demand for services. Responses from the focus groups clearly reflected the difficulty imposed on an already stretched, understaffed workforce in pivoting to rapidly address the expected surge in demand from patients related to the pandemic.

The limitations of the current study should be noted. First, the use of convenience sampling methods in this study may limit the generalizability of the findings because the perspectives provided by the participants here may not be representative of $\mathrm{CHCs}$ and their health care teams as a whole. Similarly, the majority of focus group participants were from either the Northeast or Midwest regions of the country and serve in administrative leadership roles, which may not be representative of the experiences of their regional and professional counterparts. In addition, the survey did not cover any economic or finance-related questions. Lastly, the study was not powered to detect differences in identified needs and responses based on professional role, including between administrative and clinical staff.

Nonetheless, the findings presented here offer a first look at the nature of this challenge and the needs that must be addressed to ensure that CHCs are able to provide an effective response to this growing public health emergency. Thus, the findings from this study have several immediate and long-term implications on policy, practice, and research. The COVID-19 pandemic is occurring at a time when $\mathrm{CHCs}$ nationwide are experiencing great economic uncertainty with hiring freezes, layoffs, and reduced patient care services, which can be exacerbated by the current public health crisis. Consequently, greater federal financial support for CHCs is needed to ensure these practices have the capacity to continue serving the most vulnerable members of society, thereby helping relieve the stress on hospitals and flatten the spread of COVID-19.

In addition, ensuring that $\mathrm{CHCs}$ are adequately staffed and resourced is of particular urgency during the current pandemic. There is the potential for an influx of health care use given the significant economic impact of COVID-19 and subsequent increase in persons becoming unemployed or uninsured, complicated by the fact that there are members of the US workforce with public-facing jobs that do not have remote or teleworking options and consequently are at higher risk for exposure. Lastly, the current pandemic presents an opportunity for learning and improvement. Future research regarding lessons learned during the current outbreak can help improve our health care system's preparedness for addressing future disease outbreaks and strengthen the capacity of CHCs to care for individuals and communities that are both most at risk and in need.

In conclusion, this article explored the readiness of CHCs to face issues related to COVID-19. Although the focus was on this new pandemic, the findings have broad relevance to health care in the United States not only as it relates to the current pandemic but with regards to future public health emergencies and crises. CHCs comprise the backbone of care to marginalized, vulnerable populations including the poor, racial/ethnic minorities, the homeless, migrant farm workers, HIV patients, and substance users, to name only a few. CHCs' readiness, or lack thereof, to provide health care and adapt to challenges from the current and future pandemics is fundamental to our ability as a nation to ensure that all people have access to the core health care services they need. As this article points out, health centers were not ready for COVID-19. Surveys have suggested that care delivery by federally qualified health centers (FQHCs) has shrunk by nearly half. The impact of this lack of readiness is apparent in the halting, ineffective response to the epidemic and its disproportionate impact on the most vulnerable people. Therefore, addressing the needs and enhancing the CHCs' capacity may strengthen health care for the most vulnerable members of society, and thereby help flatten the curve.

We are grateful to the members of the Weitzman Education team, Karen Ashley, Mandy Lamb, Patti Feeney, Ariel Porto, Stephanie Korman, Anna Rogers, and Lindsey Lehet, for organizing the Extension for Community Health Outcomes (ECHO) webinar that allowed us to recruit participants. We also thank the staff of the Weitzman ECHO team, Faaiza Manzoor and Leah Miller, for their technical support during the focus group interviews.

To see this article online, please go to: http://jabfm.org/content/ 34/Supplement/S85.full.

\section{References}

1. Statement on the second meeting of the international health regulations (2005) emergency committee regarding the outbreak of novel coronavirus (2019-nCoV). Geneva, Switzerland: World Health Organization; 2005. Available from: https://www. who.int/news/item/30-01-2020-statement-on-the- 
second-meeting-of-the-international-health-regulations(2005)-emergency-committee-regarding-the-outbreakof-novel-coronavirus-(2019-ncov).

2. WHO director-general's opening remarks at the media briefing on COVID-19-11 March 2020. Geneva, Switzerland: World Health Organization; 2020. Available from: https://www.who.int/directorgeneral/speeches/detail/who-director-general-s-openingremarks-at-the-media-briefing-on-covid-19-11-march2020.

3. Stiehl E, Shivaprakash N, Thatcher E, et al. Worksite health promotion for low-wage workers: a scoping literature review. Am J Health Promot 2018;32:359-73.

4. Shin P, Alvarez C, Sharac J, et al. A profile of community health center patients: implications for policy. Menlo Park, CA: Henry J. Kaiser Family Foundation; 2013. Available from: https://www.kff. org/medicaid/issue-brief/a-profile-of-communityhealth-center-patients-implications-for-policy/.

5. Health Resources and Services Administration. Health center program: impact and growth. 2020. Available from: https://bphc.hrsa.gov/about/healthcenterprogram/ index.html. Accessed March 15, 2020.

6. Information for healthcare professionals about coronavirus (COVID-19). Geneva, Switzerland: World Health Organization; 2020.
7. Krueger RA, Casey MA. Focus groups: a practical guide for applied research. 3rd ed. Thousand Oaks, CA: Sage; 2000.

8. Krueger RA, Casey MA. Focus groups: a practical guide for applied research. 5th ed. Thousand Oaks, CA: Sage; 2014.

9. Krueger RA. Focus groups: a practical guide for applied research. 2nd ed. Thousand Oaks, CA: Sage; 1994.

10. Corbin J, Strauss A. Basics of qualitative research: techniques and procedures for developing grounded theory. Thousand Oaks, CA: Sage; 2014.

11. Rust G, Satcher D, Fryer GE, Levine RS, Blumenthal DS. Triangulating on success: innovation, public health, medical care, and cause-specific US mortality rates over a half century (1950-2000). Am J Public Health 2010;100 Suppl 1:S95-S104.

12. Ablah E, Umansky E, Wilcox EA, Usher J, Church $\mathrm{J}$, Barnes V. Innovative recruitment strategies for a comprehensive worksite wellness initiative. Health Educ Res 2019;34:569-77.

13. Staffing the Safety Net: Building the Primary Care Workforce at America's Health Centers. Bethesda, MD: National Association of Community Health Centers; 2016. Available from: https://www.nachc.org/ wp-content/uploads/2015/10/NACHC_Workforce_ Report_2016.pdf. 


\section{Appendix 1. Weitzman Institute COVID-19} Needs Assessment Survey

The Weitzman Institute wants to be responsive to the needs of FQHCs throughout the United States by providing relevant supports to aid in preparation for COVID-19.

Please complete this brief survey to help us understand how we can best support you and your organization.

Thank you!

1. Please provide your name and email address

Name

E-mail Address

2. What topics do you want to learn more about to help prepare you for COVID-19? [Select all that apply]

- COVID-19 infection prevention and control

- Clinical features and epidemiologic risk of patients in the U.S. who should be evaluated for COVID-19

- Screening, diagnostic testing, and management of patients

- Processes for reporting persons under investigation (PUIs)

- Evaluation and management of close contacts of PUIs

- Safety precautions for patient-facing health care personnel

- Psychosocial impact of COVID-19 (eg, fear, panic, stigmatization, social exclusion)

- Other (please specify):

3. What topics does your organization need help with to prepare for COVID-19? [Select all that apply]

- Policies and practices for minimizing exposure to respiratory pathogens causing COVID-19 before, upon, and throughout patient visit

- Adherence to standard, contact, and airborne precautions

- Management of visitor access and movement within the facility

- Implementation of engineering controls to reduce or eliminate exposures

- Monitoring and management of ill and exposed health care personnel

- Training and education of health care personnel

- Implementation of environmental infection control procedures

- Effective communications for alerting key facility staff about known or suspected COVID-19 patients

- Processes for reporting to public health authorities

- Leveraging state and local public health agency resources

- Implementation of remote staffing planning

- Other (please specify):

4. What tools would be most helpful for you and your organization to prepare for COVID-19?

- Examples of safety planning checklists, including policies and procedures for emergency preparedness

- Virtual best practices group on COVID-19

- Webinars on how to prepare for and respond to COVID-19
- Links to trusted electronic resources

- Other (please specify):

5. What best practices at your organization would you like to share with other FQHCs to prepare for and prevent the spread of COVID-19?

6. The Weitzman team would like to invite you to participate in a related focus group in the next couple of weeks. Please let us know if you would be interested in participating.

(Note: your decision to participate has no bearing on your participation in Weitzman's education offerings):

- I may be interested in participating in a focus group regarding community health centers' response to COVID-19.

- I do not wish to participate in a focus group regarding community health centers' response to COVID-19.

\section{Appendix 2. A Multisite Study of Community Health Centers' Readiness to Address COVID-19 in the United States}

\section{Rationale}

The purpose of the focus groups is to understand, from the participants' perspective, the (1) organizational and individual strengths and gaps in preparedness, (2) role of technology, particularly telehealth, and (3) secondary consequences, including psychosocial impact, of COVID-19.

\section{Organizational and Individual Strengths and Gaps in Preparedness}

Current Organizational Policies and Procedures

What current policies and procedures does your health center have in place to address emergencies (eg, bioterrorism, disease outbreaks, natural disasters)?

Probe about understanding of related infrastructure, communications, precautions, training and education, safety.

What are the barriers and facilitators to implementing the described policies and procedures?

Probe about both internal and external factors that support/hinder implementation of their respective bealth centers' plans for responding adequately to emergencies.

Do you have any concerns about your health center's decision making during emergency events? Specifically to COVID-19?

Probe about potential difficulties around decision making including ownership, management direction, cooperative efforts and decision making, disagreements over policy, responsibility, and management response.

Organizational and Individual Preparedness for COVID-19

To date, what measures has your health center taken to specifically prepare for COVID-19?

Probe about activities that bealth center has adopted, implemented, and/or planned specifically in preparation for COVID-19. 
To what extent do you, as a provider, feel equipped and prepared for COVID-19?

Probe about understanding of health care guidance around COVID-19 including prevention and control, screening/diagnostic testing/management, processes for reporting persons under investigation (PUIs), evaluation and management of close contacts of PUIs, safety precautions.

\section{Role of Technology (Particularly Telehealth)} (15 Minutes)

\section{Role of Technology}

To what extent have technological advances improved capacity to prepare for and respond to COVID-19 at the health care systems level? Your specific health center? Your role?

Probe about potential improvements around communications across the field, staff, and patients. Probe about the role of technology today (2020) compared with prior emergencies (eg, anthrax and bioterrorism in early 2000s).

\section{Use of Telehealth}

[For participants from health centers using telehealth]: For those of you who use any form of telehealth at your health center, what forms of telehealth do you use? How does telehealth fit into your strategy for combatting COVID-19?
Probe about types of telehealth used (eg, eConsults, live telehealth, telephone patient consultation, remote patient monitoring, Project ECHO).

\section{Secondary Consequences of COVID-19}

Impact on Health System and Health Workers

What are your concerns, if any, around the psychosocial impact of COVID-19 and related efforts, on the health system? On health workers?

Probe about potential secondary consequences of COVID-19 and related efforts, including health workers' mental health (eg, stress, trauma), bealth systems' capacity to respond?

Impact on Patients

What are your concerns, if any, around the psychosocial impact of COVID-19 and related efforts on patients?

Probe about concerns around fear and isolation of the sick and quarantined; breakdown of social support structures (eg, social isolation); stigmatization of those with COVID-19; discrimination and marginalization of Asian Americans.

Closing

Is there anything else you would like to share that I haven't already asked?

Thank participant for his/her time. 